\title{
BMJ
}

\section{Outcomes of chest compression only CPR versus conventional CPR conducted by lay people in patients with out of hospital cardiopulmonary arrest witnessed by bystanders: nationwide population based observational study}

Toshio Ogawa, assistant professor,, Manabu Akahane, lecturer, ${ }^{1}$ Soichi Koike, associate professor, ${ }^{2}$ Seizan Tanabe, professor, ${ }^{3}$ Tatsuhiro Mizoguchi, specialist for ambulance service, ${ }^{4}$ Tomoaki Imamura, professor ${ }^{1}$

${ }^{1}$ Nara Medical University School of Medicine, Department of Public Health, Health Management and Policy, 840 Shijo-cho, Kashihara, Nara 634-8521, Japan

${ }^{2}$ The University of Tokyo Hospital, Department of Planning, Information and Management, 7-3-1 Hongo, Bunkyo-ku, Tokyo 113-8655, Japan ${ }^{3}$ Foundation for Ambulance Service Development, Emergency Life-Saving Technique Academy of Tokyo, 4-5 Minamiosawa, Hachioji, Tokyo 192-0364, Japan ${ }^{4}$ Fire and Disaster Management Agency, Ambulance Service Planning Office, 2-1-2

Kasumigaseki, Chiyoda-ku, Tokyo 100-8927, Japan

Correspondence to: T Ogawa ogawat@naramed-u.ac.jp

Cite this as: BMJ 2011;342:C7106 doi:10.1136/bmi.c7106

\section{ABSTRACT}

Objective To compare the effectiveness of cardiopulmonary resuscitation (CPR) with chest compression only and conventional CPR on outcomes after cardiopulmonary arrest out of hospital.

Design Nationwide population based observational study.

Setting A nationwide emergency medical service system in Japan.

Population All consecutive patients with out of hospital cardiopulmonary arrest, January 2005 to December 2007 in Japan, witnessed at the moment of collapse. Lay people attempted chest compression only CPR $(n=20707)$ or conventional CPR (mouth to mouth ventilation and chest compression) $(n=19328)$, and patients were transferred to hospital by ambulance.

Main outcome measures Factors associated with better outcomes (assessed with $\chi^{2}$, multiple logistic regression analysis, odds ratios and their $95 \%$ confidence intervals): one month survival and neurologically favourable one month survival rates defined as category one (good cerebral performance) or two (moderate cerebral disability) of the cerebral performance categories.

Results Conventional CPR was associated with better outcomes than chest compression only CPR, for both one month survival (adjusted odds ratio 1.17, 95\% confidence interval 1.06 to 1.29$)$ and neurologically favourable one month survival $(1.17,1.01$ to 1.35$)$. Neurologically favourable one month survival decreased with increasing age and with delays of up to 10 minutes in starting CPR for both conventional and chest compression only CPR. The benefit of conventional CPR over chest compression only CPR was significantly greater in younger people in non-cardiac cases $(P=0.025)$ and with a delay in start of CPR after the event was witnessed in non-cardiac cases $(P=0.015)$ and all cases combined $(P=0.037)$.

Conclusions Conventional CPR is associated with better outcomes than chest compression only CPR for selected patients with out of hospital cardiopulmonary arrest, such as those with arrests of non-cardiac origin and younger people, and people in whom there was delay in the start of CPR.

\section{INTRODUCTION}

The survival rate in patients with cardiopulmonary arrest out of hospital is generally low, ${ }^{1}$ though some patients can recover after immediate and appropriate resuscitation ${ }^{2}$ involving early access to emergency care, early cardiopulmonary resuscitation (CPR), early defibrillation, and early advanced cardiovascular life support ${ }^{3}$ - a series of events known as the "chain of survival." ${ }^{4}$ In 2005 the International Liaison Committee on Resuscitation (ILCOR) achieved a consensus for out of hospital resuscitation, ${ }^{5}$ indicating that the most important determinant of survival from sudden cardiac arrest is the presence of a trained rescuer who is ready, willing, able, and equipped to act.

Bystander CPR entails mouth to mouth ventilation (rescue breathing) and chest compression, known as conventional CPR, but recently chest compression only CPR has become more popular. Some people are concerned about conducting rescue breathing because of the risk of infectious diseases or unwillingness to perform mouth to mouth breathing for religious or other reasons. ${ }^{67}$ The SOS-KANTO study group ${ }^{8}$ recently reported that it is possible to maintain the partial pressure of oxygen $\left(\mathrm{PaO}_{2}\right)$ for about 4-10 minutes after cardiac arrest and that there is better cerebrovascular circulation with chest compression only CPR than with conventional CPR. Also, several studies have reported that recovery with chest compression only CPR is similar to ${ }^{9-11}$ or better than recovery with conventional CPR. ${ }^{8}$

The latest guidelines for CPR and emergency cardiovascular care from the American Heart Association (AHA), published in 2005, advise that lay people should be encouraged to do compression only CPR if they are unable or unwilling to provide rescue breathing, although the best method is conventional CPR. ${ }^{12}$ In 2008, an AHA science advisory committee 
recommended that a bystander who has been trained in CPR and is confident to provide rescue breaths should provide either conventional CPR or hands only CPR, which means more emphasis on the application of chest compression only CPR. ${ }^{13}$ Consequently, chest compression only CPR has become a popular technique of bystander resuscitation. As mentioned above and as pointed out by Steen, ${ }^{14}$ however, the choice of immediate resuscitation technique implemented by bystanders is still controversial.

We compared the effectiveness of conventional CPR and chest compression only CPR using a large national database of out of hospital cardiopulmonary arrest in Japan.

\section{METHODS}

Data source

In Japan, a single emergency network with ambulance services covers the whole country and is administered by the Fire and Disaster Management Agency (FDMA). Calls to the universal emergency access number 119 are directly connected to a dispatch centre located in the regional fire defence headquarters, covering 807 fire stations as of 2007. On receipt of a call, the nearest available ambulance is sent to the incident. The agency supervises the emergency medical service system throughout the country.

This nationwide population based observational study covered consecutive patients with out of hospital cardiopulmonary arrest in Japan from 2005 to 2007. We entered 318141 cases on a national level database developed by the Fire and Disaster Management Agency. All patients in this database experienced cardiopulmonary arrest outside medical facilities and were transferred to hospitals. In the database they were classified as cardiopulmonary arrest confirmed by the emergency medical service on arrival at the incident, cardiopulmonary arrest in an ambulance during transfer to hospital, or supposed cardiopulmonary arrest in which the patient had already been resuscitated when the emergency medical service arrived. As there is only one nationwide system of ambulance service in Japan, the database in the present study involved all cases of out of hospital cardiopulmonary arrest in which the patient was transported to hospital.

The data on out of hospital cardiopulmonary arrest were gathered by the emergency medical service in the local fire departments from their observation and from interviews with bystanders and physicians in charge of the patients. Data were obtained on age, sex, whether the collapse was witnessed, whether bystander CPR was performed, the category of bystander (such as lay person or emergency medical service staff), whether the dispatcher gave assistance by telephone, cause of cardiac arrest such as cardiac or non-cardiac origin, and initial identified cardiac rhythm. In collaboration with the physicians in charge, emergency medical service staff also obtained outcome data such as survival and category of cerebral performance ${ }^{15}$ a month after hospital admission. The physician in charge, in collaboration with emergency medical service staff, determined the cause of cardiac arrest clinically. Emergency medical service staff also interviewed bystanders on site to determine the length of time from collapse to the first resuscitation attempt and identified the initial cardiac rhythm.

Emergency medical service staff entered all information at local fire departments using an online entry form, which basically conformed to the Utstein form ${ }^{16}$ with some additions. The data were verified by emergency medical service staff, anonymised at the local fire department, and transferred and stored on the database at the Fire and Disaster Management Agency. The database was checked by the computer system and compiled for public use by the agency.

We analysed this anonymous database with permission from the agency. According to the informed consent guidelines in Japan, ${ }^{17}$ it is unnecessary to have informed consent from each patient to use secondary data such as on this database.

\section{Inclusion and exclusion criteria}

Cases comprised people with out of hospital cardiopulmonary arrests of cardiac and non-cardiac origin - such as stroke, respiratory obstruction, or traumawitnessed by lay people where CPR was provided by bystander(s) (fig 1). Witnesses could include family members, friends, colleagues, passersby, and others. Among the total cases in the database $(n=318141)$, we excluded the 190646 that were not witnessed. We also excluded the 25521 cases witnessed by fire services staff, emergency specialists, and emergency and rescue specialists and 193 cases witnessed by unknown bystanders. Our final group for inclusion therefore comprised 101781 cases of out of hospital cardiopulmonary arrest witnessed by lay people.

We classified bystander CPR as chest compression only CPR or conventional CPR (both chest compression and rescue breathing). Among the 101781 cases,

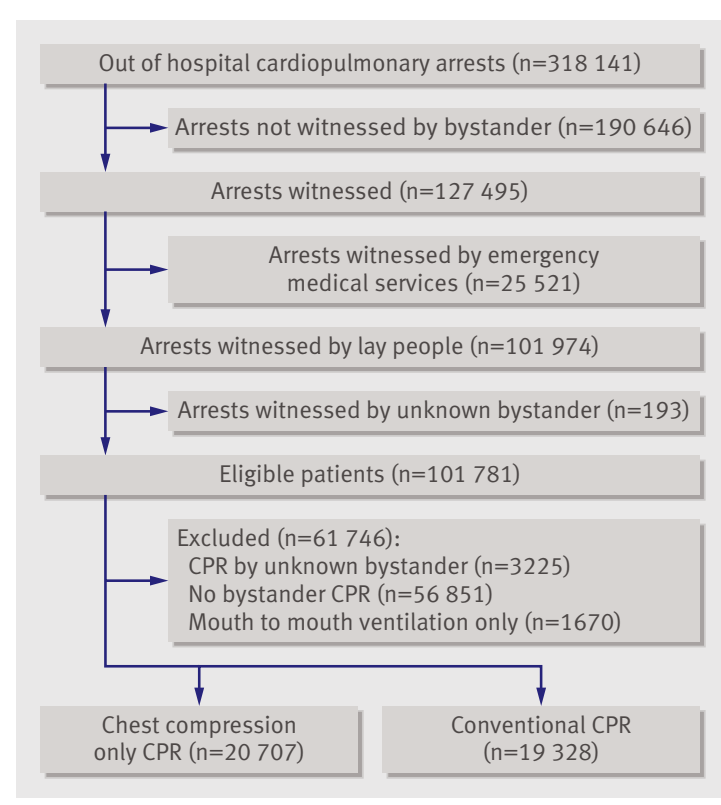

Fig 1 1 Study profile with selection of participants 
we excluded from our analysis cases with no bystander CPR ( $\mathrm{n}=56851$ ), cases with rescue breathing only $(\mathrm{n}=1670)$, and cases with CPR from an unknown bystander $(\mathrm{n}=3225)$. We included 20707 cases with chest compression only CPR and 19328 cases with conventional CPR in the present study. Table 1 summarises the cases analysed.

\section{Statistical analysis}

Our study outcomes were rates of one month survival and neurologically favourable one month survival,

\begin{abstract}
Table 1 Characteristics of study participants with out of hospital cardiopulmonary arrest witnessed by bystander, Japan 2005-7. Figures are numbers (percentages) unless stated otherwise. Base number supplied when this differs from total
\end{abstract}

\begin{tabular}{|c|c|c|c|}
\hline & $\begin{array}{l}\text { Chest compression only } \\
\text { CPR }\end{array}$ & $\begin{array}{l}\text { Conventional } \\
\quad \text { CPR }\end{array}$ & $\begin{array}{c}\text { P for } \\
\text { difference }\end{array}$ \\
\hline No of cases & 20707 & 19328 & - \\
\hline Men & $12714(61.4)$ & $10698(55.3)$ & $<0.001$ \\
\hline \multicolumn{4}{|l|}{ Age group (years): } \\
\hline$<20$ & $277 / 20706(1.3)$ & 482/19 324 (2.5) & \multirow{6}{*}{$<0.001$} \\
\hline $20-39$ & $770 / 20706(3.7)$ & $768 / 19324(4.0)$ & \\
\hline 40-59 & 2717/20 $706(13.1)$ & 2602/19 324 (13.5) & \\
\hline $60-79$ & $8182 / 20706(39.5)$ & 6836/19 $324(35.4)$ & \\
\hline 80-99 & $8667 / 20706$ (41.9) & 8505/19324 (44.0) & \\
\hline$\geq 100$ & $93 / 20706(0.4)$ & $131 / 19324(0.7)$ & \\
\hline Average age (years) & 73.1 & 72.7 & $0.030^{\star}$ \\
\hline $\begin{array}{l}\text { Average time from event witnessed to } \\
\text { bystander CPR (min) }\end{array}$ & $4.1(n=19558)$ & $3.0(n=18294)$ & $<0.001^{\star}$ \\
\hline \multicolumn{4}{|c|}{ Duration from event witnessed to bystander CPR ( $\mathrm{min}$, up to $10 \mathrm{~min}$ ): } \\
\hline 0 & 5763/17931 (32.1) & $7047 / 17480(40.3)$ & \multirow{6}{*}{$<0.001$} \\
\hline $1-2$ & 5287/17931 (29.5) & $5414 / 17480(31.0)$ & \\
\hline $3-4$ & 2717/17931 (15.2) & $1956 / 17480(11.2)$ & \\
\hline $5-6$ & 1694/17 931 (9.4) & $1328 / 17480(7.6)$ & \\
\hline 7-8 & 1616/17931 (9.0) & $1090 / 17480(6.2)$ & \\
\hline $9-10$ & $854 / 17931(4.8)$ & $645 / 17480(3.7)$ & \\
\hline \multicolumn{4}{|l|}{ Category of bystander/s: } \\
\hline Family members & $12844(62.0)$ & $9017(46.7)$ & \multirow{5}{*}{$<0.001$} \\
\hline Friends & $932(4.5)$ & $935(4.8)$ & \\
\hline Colleagues & $728(3.5)$ & $617(3.2)$ & \\
\hline Passersby & $876(4.2)$ & $583(3.0)$ & \\
\hline Other lay people & $5327(25.7)$ & $8176(42.3)$ & \\
\hline Dispatcher assisted with CPR & 12 308/20 695 (59.5) & $9613 / 19248(49.9)$ & $<0.001$ \\
\hline \multicolumn{4}{|l|}{ Cause of arrest: } \\
\hline Cardiac origin & $10729 / 19638(54.6)$ & 9929/18 $022(55.1)$ & \multirow{6}{*}{$<0.001$} \\
\hline Non-cardiac, cerebrovascular diseases & 1086/19 $638(5.5)$ & 1052/18 $022(5.8)$ & \\
\hline Non-cardiac, respiratory diseases & $1559 / 19638(7.9)$ & $1464 / 18022(8.1)$ & \\
\hline Non-cardiac, malignant tumours & $780 / 19638(4.0)$ & $480 / 18022(2.7)$ & \\
\hline Non-cardiac, external causes & 3000/19 $638(15.3)$ & 2685/18 $022(14.9)$ & \\
\hline Non-cardiac, others & 2484/19 $638(12.6)$ & 2412/18 $022(13.4)$ & \\
\hline VF/VT as first documented rhythm & $3412(16.5)$ & $3266(16.9)$ & 0.260 \\
\hline Drug use during advanced CPR & $906 / 20703(4.4)$ & $731 / 18959(3.9)$ & 0.009 \\
\hline AED by bystander & $120 / 20706(0.6)$ & $383 / 18692(2.0)$ & $<0.001$ \\
\hline First shock by EMS & $4256 / 20683(20.6)$ & 4037/19 $143(21.1)$ & 0.209 \\
\hline $\begin{array}{l}\text { Average time from event witnessed to CPR } \\
\text { by EMS (min) }\end{array}$ & $12.7(n=19970)$ & $13.1(n=18458)$ & $<0.001^{\star}$ \\
\hline $\begin{array}{l}\text { Average time from event witnessed to } \\
\text { arrival at hospital (min) }\end{array}$ & 35.7 (20 574) & $36.3(19184)$ & $<0.001^{*}$ \\
\hline
\end{tabular}

defined as category one (good cerebral performance) or two (moderate cerebral disability) of the cerebral performance categories. ${ }^{15}$

We analysed neurologically favourable one month survival in people with events witnessed by lay people who provided either conventional CPR or chest compression only CPR based on 20 year age categories ( 0 $19,20-39,40-59,60-79,80-99$, and $>100)$, the duration between the time of being witnessed by a bystander and a bystander starting CPR in 2 minute intervals up to 10 minutes (0, 1-2, 3-4, 5-6, 7-8, and 9-10), and origin of cardiac arrest (cardiac or non-cardiac origin). We excluded 20 people in whom age was unknown from the age analysis. We also excluded from the duration analysis 3237 people in whom there was more than 10 minutes between the time of being witnessed by a bystander and a bystander starting CPR and 1387 in whom the duration was unknown.

We used $\chi^{2}$ for analysis and calculated odds ratios and $95 \%$ confidence intervals to assess significance, defined as $\mathrm{P}<0.05$. Independent sample $t$ tests were conducted to compare the average of the samples. We used multiple logistic regression analysis to assess the factors associated with better outcomes and calculated adjusted odds ratios and their 95\% confidence intervals. Interactions between types of CPR and age or duration categories were assessed in a multiadjusted interaction model adjusted for potential confounding factors.

Potential confounding factors were selected by clinical considerations. These included age, sex, assistance from dispatcher, initial identified cardiac rhythm, cause of cardiac arrest (cardiac or non-cardiac origin), the relation of the bystander to the patient (family member, colleagues, etc), use of a public access automated external defibrillator to administer a first shock, first shock from emergency medical staff, the use of a drug during CPR, duration between bystander witnessing event to bystander starting CPR, duration between bystander witnessing event to CPR by emergency medical staff, and duration between bystander witnessing event to patient's arrival at hospital. We carried out principal components analyses to confirm the clinical considerations. Selected confounding factors were used for the multivariate analyses. All statistical analyses were conducted with PASW v 18 (SPSS, Chicago, IL).

\section{RESULTS}

\section{Comparison of outcomes}

The one month survival rates of patients in cases with conventional CPR were significantly higher than those with chest compression only CPR (adjusted odds ratio $1.17,95 \%$ confidence interval 1.06 to 1.29$)$. Neurologically favourable one month survival was also significantly higher with conventional CPR than with chest compression only CPR (1.17, 1.01 to 1.35$)$, showing a similar tendency to the one month survival rate (table 2). 
Table 2 | One month survival and neurologically favourable one month survival in cases of out of hospital cardiopulmonary arrest witnessed by bystander with chest compression only CPR and conventional CPR. Figures are percentages (numbers of participants)

\begin{tabular}{|c|c|c|c|c|}
\hline & \multirow[b]{2}{*}{ Chest compression only CPR } & \multirow[b]{2}{*}{ Conventional CPR } & \multicolumn{2}{|c|}{ Odds ratio $(95 \% \mathrm{Cl})$, $\mathrm{P}$ value } \\
\hline & & & Unadjusted & Adjusted* \\
\hline One month survival & $8.7(1799 / 20707)$ & $10.3(1997 / 19327)$ & 1.21 (1.13 to 1.29$),<0.001$ & 1.17 (1.06 to 1.29 ), 0.002 \\
\hline Neurologically favourable one month survival & $4.6(943 / 20662)$ & $5.6(1070 / 19247)$ & 1.23 (1.12 to 1.35$),<0.001$ & 1.17 (1.01 to 1.35$), 0.037$ \\
\hline
\end{tabular}

*Adjusted for age, sex, assistance from dispatcher, initial identified cardiac rhythm, cause of cardiac arrest, relation of bystander to patient, use of public access automated external

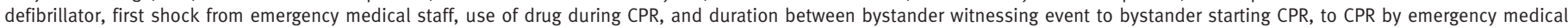
staff, and to patient's arrival at hospital.

Effectiveness of conventional CPR and chest compression only CPR by age

Rates of neurologically favourable one month survival decreased with increasing age for both conventional and chest compression only CPR. The benefit of conventional CPR over chest compression only CPR was significantly greater at younger ages for non-cardiac cases $(\mathrm{P}=0.025)$ but similar at all ages for cardiac cases $(\mathrm{P}=0.109)$ and all cases combined $(\mathrm{P}=0.951)$ (fig 2$)$.

Effectiveness of conventional CPR and chest compression only CPR by time to CPR

Rates of neurologically favourable one month survival decreased with delay in start of CPR for both conventional and chest compression only CPR. The benefit of conventional CPR over chest compression only CPR was significantly increased with the length of delay before start of CPR for non-cardiac cases $(\mathrm{P}=0.015)$ and all cases combined $(\mathrm{P}=0.037)$ but was similar for cardiac cases $(\mathrm{P}=0.369)$ (fig 3).

\section{DISCUSSION}

In people who experience an out of hospital cardiac arrest witnessed by a bystander, conventional CPR (that is, mouth to mouth ventilation and chest compression) is associated with better rates of one month survival and neurologically favourable one month survival than chest compression only CPR.

\section{Comparisons with other studies}

Our findings differ from those of previous studies, which showed similar outcomes between the two methods of $\mathrm{CPR}^{9-11}$ or showed that chest compression only CPR had a better outcome. ${ }^{8}$ These differences could be explained by sample size. We analysed over 40000 cases of out of hospital cardiac arrest in which the patient received either conventional or chest compression only CPR. All events were witnessed by lay bystanders. Cases were sampled from all cases of out of hospital cardiopulmonary arrest in Japan in a three year period. This is a larger sample compared with previous studies in Japan, ${ }^{810}$ Sweden, ${ }^{9}$ Norway, ${ }^{11}$ and Singapore ${ }^{18}$ and provided greater statistical power. In addition, as all consecutive cases of out of hospital cardiopulmonary arrest in Japan were included in the database, selection bias was minimal.

The better outcomes with conventional CPR compared with chest compression only CPR were derived mainly from cases of non-cardiac origin at younger ages. With regard to outcomes for younger patients,
Kitamura et al analysed those aged under 17 using the same database and found consistent results. ${ }^{19}$ In our study there were only $277(1 \%)$ patients aged under 20 in the chest compression only CPR group and $482(3 \%)$ in the conventional CPR group (table 1). Among this younger group, 48\% (223/465) of arrests of non-cardiac origin were caused by external causes, including drowning, trauma, asphyxia, and drug addiction, in which conventional CPR was associated with a significantly higher rate of neurologically favourable one month survival $(26 \%, 34 / 131)$ than
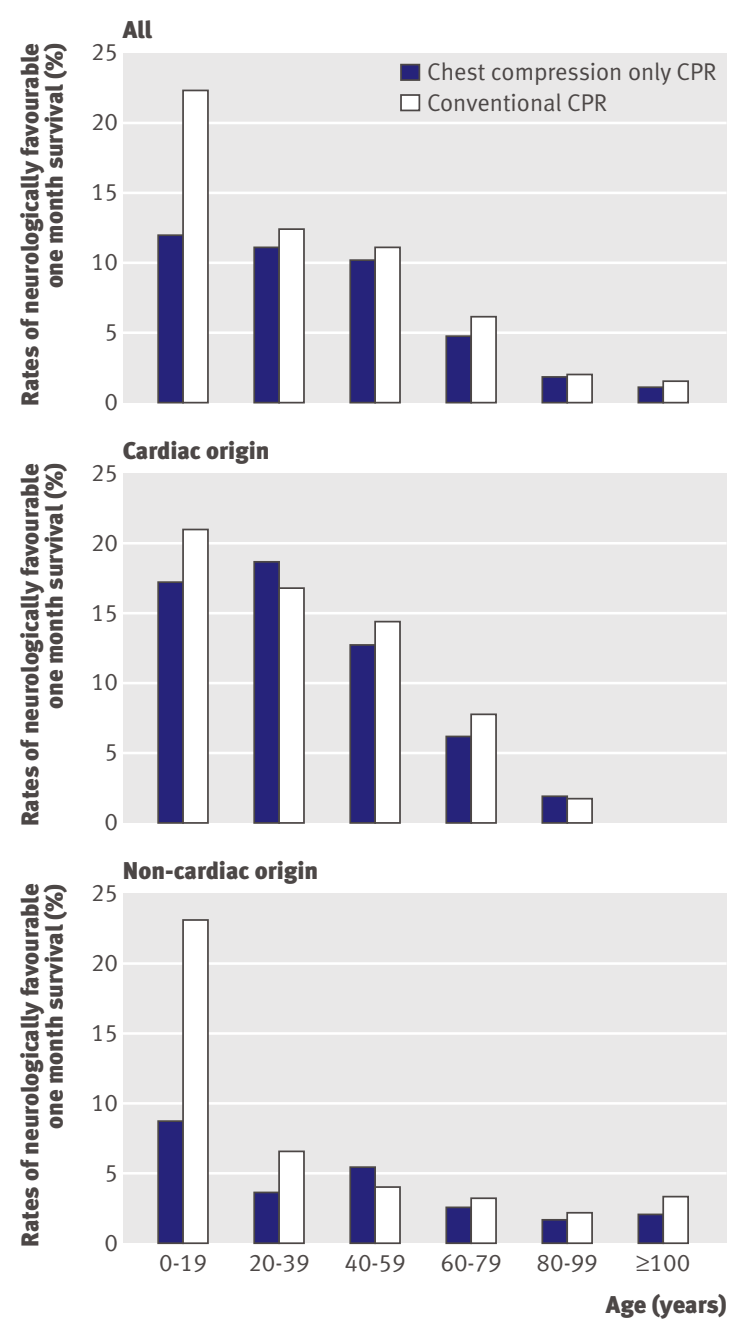

$\overline{\text { Fig } 2 \text { | Rates of neurologically favourable one month survival }}$ after chest compression only CPR and conventional CPR in people with out of hospital cardiopulmonary arrest witnessed by bystander by age and origin of cardiac arrest 
chest compression only CPR $(7 \%, 6 / 91)$. This finding might have influenced the better outcomes with conventional CPR than with chest compression only CPR at younger ages.

With regard to older patients, Iwami et $\mathrm{al}^{10}$ and the SOS-KANTO study group ${ }^{8}$ selected events of cardiac origin in patients aged over 18 . Our results are mostly in agreement with their results in that there was no difference between conventional and chest compression only CPR in cases of arrest with cardiac origin. Hupfl et al carried out a meta-analyses of studies in adult patients; these showed that chest compression only CPR was associated with outcomes better than or similar to conventional $\mathrm{CPR} .{ }^{20} \mathrm{~A}$ possible reason for the difference in results from the present study could be that there were different inclusion/exclusion criteria between the studies. We included all age groups and causes, whereas Hupfl et al included only adults, and some studies in their analysis involved only cardiac cases. $^{20}$

Conventional CPR was associated with better outcomes than chest compression only CPR for patients with delays of up to 10 minutes in starting CPR after the event was witnessed for cases of non-cardiac origin and for all cases combined (fig 3). The benefit of conventional CPR for all cases combined was mainly derived from cases with non-cardiac origin. These results are different from those of the SOS-KANTO study, which showed that outcome was better with chest compression only CPR than with conventional CPR given within four minutes of arrest. ${ }^{8}$ A possible reason for this difference could be the difference of inclusion/exclusion criteria for these two studies. We included all age groups, whereas the SOS-KANTO study involved only adults.

\section{Strengths and limitations of study}

Our findings suggest that new guidelines are needed for bystander CPR. We recommend that conventional CPR should be used in younger patients with an arrest of non-cardiac origin and in all patients with an arrest of non-cardiac origin in whom start of CPR is delayed. It might be difficult to apply these recommendations directly to future guidelines for several reasons. People need to have undergone sufficient training to provide effective conventional CPR. It is also difficult to assess the causes of cardiac arrest, particularly for lay people, it is difficult to assess the time elapsed since the cardiac arrest, and there are well known barriers to conducting rescue breathing. ${ }^{6721}$ It might therefore be better to recommend conventional CPR under certain circumstances only for bystanders with suitable experience or for those who have had training in conventional CPR.

This study has some limitations. Firstly, the database we used might have included patients without cardiopulmonary arrest. This is because it would have been difficult for lay people to judge whether the patients were in cardiopulmonary arrest in an emergency situation. Therefore, our results could have overestimated the effect of CPR. Although further assessment of the selection criteria might be needed, we believe that it
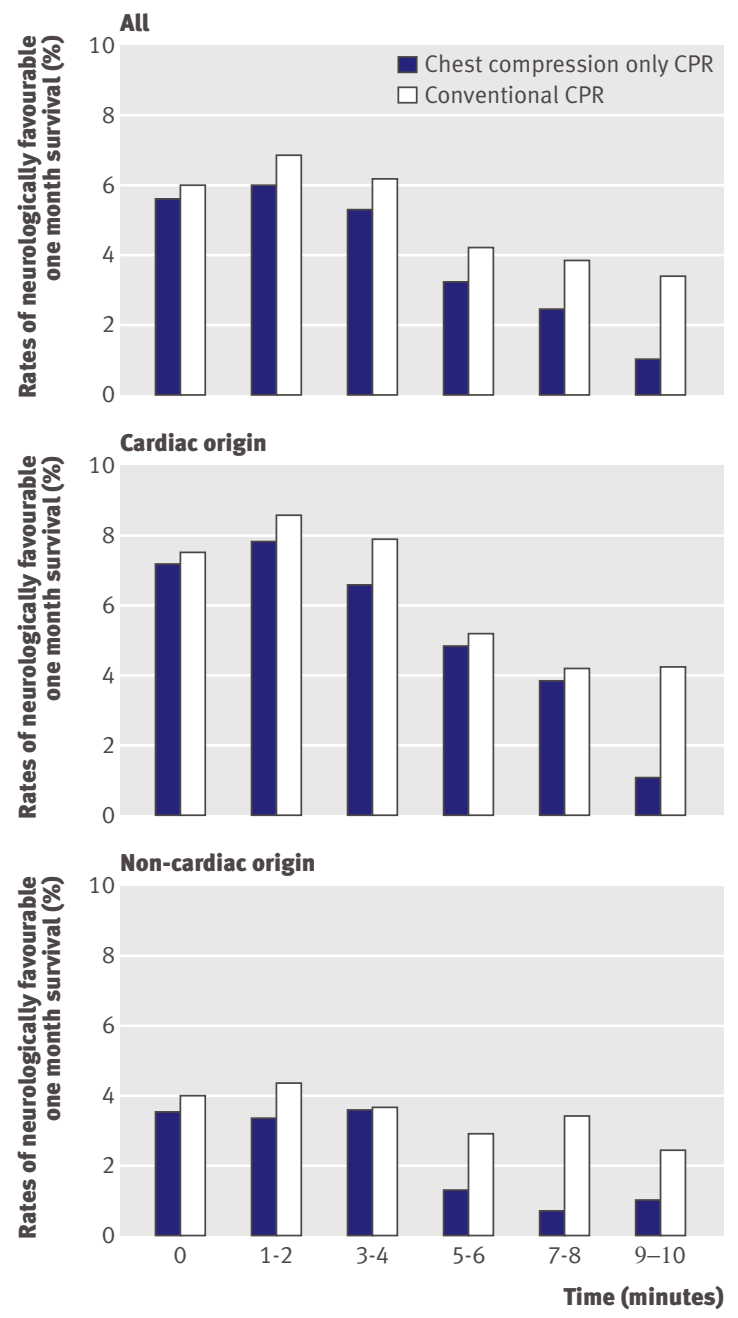

Fig 3 Rates of neurologically favourable one month survival after chest compression only CPR and conventional CPR in people with out of hospital cardiopulmonary arrest witnessed by bystander by time between event and start of CPR by bystander and origin of cardiac arrest

did not affect our results because this was a comparative study between conventional CPR and chest compression only CPR and any inclusion errors would be distributed equally between the two methods. Secondly, though we analysed the data by age, there were few cases in the lower age groups, particularly those under 40. Therefore, there was a risk of lower accuracy and higher unpredicted confounding factors for the analysis of patients aged under 40. Thirdly, the times, in particular the time the event was witnessed, might have been inaccurate. This was reported by the bystander, but it might have been difficult to identify the exact time of onset of the event in such an emergency situation. Fourthly, the selection of confounding factors needs to be considered further. We selected these factors based on clinical considerations with principal components analyses to confirm the selection. As the results of the statistical technique were the same as the results of the clinical considerations, we used the selected confounding factors. We cannot be sure, however, that we included all potential confounding 


\section{WHAT IS ALREADY KNOWN ON THIS TOPIC}

Although cardiopulmonary resuscitation (CPR) with chest compression only has become popular for bystander resuscitation, its effect compared with conventional CPR (with mouth to mouth ventilation) in patients with out of hospital cardiopulmonary arrest is still controversial

\section{WHAT THIS STUDY ADDS}

Conventional CPR is associated with better outcomes than chest compression only CPR for one month survival and neurologically favourable one month survival in patients with out of hospital cardiopulmonary arrest

The better outcomes were seen in younger patients with events of non-cardiac origin and in patients with a delay of up to 10 minutes to the start of CPR after the event was witnessed for non-cardiac cases and all cases combined
Ethical approval: This study was approved by the ethics committee of Nara Medical University (authorisation code: 260). Patient consent was not required for use of secondary data.

Data sharing: No additional data available.

1 Rea TD, Eisenberg MS, Sinibaldi G, White RD. Incidence of EMS treated out-of-hospital cardiac arrest in the United States. Resuscitation 2004:63:17-24

2 Holmberg M, Holmberg S, Herlitz J. The problem of out-of-hospital cardiac-arrest prevalence of sudden death in Europe today. Am J Cardiol 1999:83:88-90D.

3 Vukmir RB. Witnessed arrest, but not delayed bystander cardiopulmonary resuscitation improves prehospital cardiac arrest survival. Emerg Med / 2004:21:370-3.

4 Cummins RO, Ornato JP, Thies WH, Pepe PE. Improving survival from sudden cardiac arrest: the "chain of survival" concept. A statement for health professionals from the Advanced Cardiac Life Support Subcommittee and the Emergency Cardiac Care Committee, American Heart Association. Circulation 1991;83:1832-47.

5 Proceedings of the 2005 International Consensus on Cardiopulmonary Resuscitation and Emergency Cardiovascular Care Science with Treatment Recommendation. Resuscitation 2005;67:157-341.

6 Locke CJ, Berg RA, Sanders AB, Davis MF, Milander MM, Kern KB, et al. Bystander cardiopulmonary resuscitation. Concerns about mouth-to-mouth contact. Arch Intern Med 1995;155:938-43.

7 Taniguchi T, Omi W, Inaba H. Attitudes toward the performance of bystander cardiopulmonary resuscitation in Japan. Resuscitation 2007;75:82-7.

8 SOS-KANTO. Cardiopulmonary resuscitation by bystanders with chest compression only (SOS-KANTO): an observational study. Lancet 2007;369:920-6.

9 Bohm K, Rosenqvist M, Herlitz |, Hollenberg |, Svensson L. Suvival is similar after standard treatment and chest compression only in outof-hospital bystander cardiopulmonary resuscitation. Circulation 2007:116:2908-12.

10 Imami T, Kawamura T, Hiraide A, Berg RA, Hayashi Y, Nishiuchi T, et al. Effectiveness of bystander-initiated cardiac-only resuscitation for patients with out-of-hospital cardiac arrest. Circulation 2007:116:2900-7.

11 Olasveengen TM, Wik L, Steen PA. Standard basic life support vs continuous chest compressions only in out-of-hospital cardiac arrest. Acta Anaesthesiol Scand 2008:52:914-9.

12 AHA. 2005 American Heart Association guidelines for cardiopulmonary resuscitation and emergency cardiovascular care. Circulation 2005;112(suppl 24):IV1-203.

13 Sayre MR, Berg RA, Cave DM, Page RL, Potts J, White RD. Hands-only (compression-only) cardiopulmonary resuscitation: a call to action for bystander response to adults who experience out-of-hospital sudden cardiac arrest: a science advisory for the public from the American Heart Association Emergency Cardiovascular Care Committee. Circulation 2008;117:2162-7.

14 Steen PA. Does active rescuer ventilation have a place during basic cardiopulmonary resuscitation? Circulation 2007;116:2514-6

15 Cummins RO, Chamberlain DA, Abramson NS, Allen M, Baskett PJ, Becker $L$, et al. Recommended guidelines for uniform reporting of data from out-of-hospital cardiac arrest: the Utstein style. A statement for health professionals from a task force of the American Heart Association, the European Resuscitation Council, the Heart and Stroke Foundation of Canada, and the Australian Resuscitation Council. Circulation 1991;84:960-75.

16 Jacobs I, Nadkarni V, Bahr J, Berg RA, Billi JE, Bossaert L, et al. Cardia arrest and cardiopulmonary resuscitation outcome reports: update and simplification of the Utstein templates for resuscitation registries. A statement for healthcare professionals from a task force of the international liaison committee on resuscitation (American Heart Association, European Resuscitation Council, Australian Resuscitation Council, New Zealand Resuscitation Council, Heart and Stroke Foundation of Canada, InterAmerican Heart Foundation, Resuscitation Council of Southern Africa). Resuscitation 2004:63:233-49.

17 Ministry of Education Culture, Sports, Science and Technology of Japan/Ministry of Health, Labour and Welfare of Japan. A guideline for epidemiology studies. 2006

18 Ong ME, Ng FS, Anushia P, Tham LP, Leong BS, Ong VY, et al. Comparison of chest compression only and standard cardiopulmonary resuscitation for out-of-hospital cardiac arrest in Singapore. Resuscitation 2008;78:119-26.

19 Kitamura T, Iwami T, Kawamura T, Nagao K, Tanaka H, Nadkarni VM, et al. Conventional and chest-compression-only cardiopulmonary resuscitation by bystanders for children who have out-of-hospital cardiac arrests: a prospective, nationwide, population-based cohor study. Lancet 2010;375:1347-54 Interest form at www.icmje.org/coi_disclosure.pdf (available on request from the corresponding author) and declare: no support from any organisation for the submitted work; no financial relationships with any organisations that might have an interest in the submitted work in the previous three years; no other relationships or activities that could appear to have influenced the submitted work. 
20 Hupfl M, Selig HF, Nagele P. Chest-compression-only versus standard cardiopulmonary resuscitation: a meta-analysis. Lancet 2010;376:1552-7.

21 Caves ND, Irwin MG. Attitudes to basic life support among medical students following the 2003 SARS outbreak in Hong Kong. Resuscitation 2006;68:93-100.

22 Sayre MR, Cantrell SA, White LJ, Hiestand BC, Keseg DP, Koser S. Impact of the 2005 American Heart Association cardiopulmonary resuscitation and emergency cardiovascular care guidelines on outof-hospital cardiac arrest survival. Prehosp Emerg Care 2009;13:469-77.

23 Arrich J, Zeiner A, Sterz F, Janata A, Uray T, Richling N, et al. Factors associated with a change in functional outcome between one month and six months after cardiac arrest: a retrospective cohort study. Resuscitation 2009;80:876-80.

Accepted: 8 November 2010 